Horizons philosophiques

\title{
Épreuves de l'étranger : entretien avec Nancy Huston réalisé par Mi-Kyung YI
}

\section{Mi-Kyung Yi}

Volume 12, numéro 1, automne 2001

Langue : identité plurielle

URI : https://id.erudit.org/iderudit/801192ar

DOI : https://doi.org/10.7202/801192ar

Aller au sommaire du numéro

Éditeur(s)

Collège Édouard-Montpetit

ISSN

1181-9227 (imprimé)

1920-2954 (numérique)

Découvrir la revue

\section{Citer ce document}

Yi, M.-K. (2001). Épreuves de l'étranger : entretien avec Nancy Huston réalisé par Mi-Kyung YI. Horizons philosophiques, 12(1), 1-16.

https://doi.org/10.7202/801192ar d'utilisation que vous pouvez consulter en ligne.

https://apropos.erudit.org/fr/usagers/politique-dutilisation/ 


\title{
Épreuves de l'étranger: Entretien avec Nancy Huston réalisé par Mi-Kyung $\mathrm{YI}^{*}$
}

\author{
"Une note toute seule est dépourvue de signification, \\ donc innocente...Seul un intervalle, un accord, l'asso- \\ ciation de deux notes ou plus peut être étrange, ou \\ beau, ou perturbant. Voire diabolique". \\ Nancy Huston, Instruments des ténèbres.
}

Mi-Kyung Yi : Comme pour beaucoup de personnes, commencer est pour moi toujours une tâche difficile. À réfléchir sur le début de notre entretien, j'avais en tête deux images extraites de vos livres, qui me paraissaient illustrer de manière particulièrement éloquente la double dimension de l'origine. La première se trouve dans Trois fois septembre, évoquée par la voix de Selena; c'est celle d'un arbre solidement enraciné dans la terre, dont les branches se ramifient. Cette jeune fille à l'âme errante rêve des mots et d'un corps qui seraient à l'image de cet arbre. L'autre image est celle du piano à queue de Hölderlin dont quelques cordes sont amputées à coup de ciseaux. Voilà deux images saisissantes évocatrices de la double tension qui me semble traverser vos livres.

Nancy Huston : Le piano à queue de Hölderlin, c'est dans quel livre?

MK.Y. : C'est dans Instruments des ténèbres. Je suis ravie de vous l'apprendre! (rires).

N.H. : Oui, je me souviens très bien de ce piano. En plus d'être le violon scordatura, c'est-à-dire désaccordé, l'héroïne s'identifiait à ce piano dont Hölderlin avait coupé quelques cordes de sorte qu'en jouant, l'on ne savait jamais si on allait produire un son ou non. Et il ne faut pas oublier aussi que les pianos sont faits avec des arbres. Dans une pièce radiophonique que j'ai écrite, au début de ma deuxième grossesse, automne 97 , il y a un rapprochement, une comparaison, entre le piano et l'arbre. À la fin de la pièce, on fracasse un piano à queue avec une hache. On prend la hache, on abat le piano comme si c'était un arbre. C'est intéressant que vous ayez choisi ces deux images séparées, alors que quelque part, elles se trouvent ensemble aussi. Et l'arbre : il y a un certain nombre de métaphores autour de l'arbre que j'utilise, souvent, pour expliquer ma trajectoire. J'ai eu une maladie neurologique; j'en parle dans Journal de la création - justement à la sortie de Lettres parisiennes. Histoires d'exil, comme fait

* Mi-Kyung Yi, est psychanalyste, chargée de cours à l'Université Paris 7, et auteure de Herméneutique et psychanalyse... si proches, si étrangères, Paris, PUF, 2001. 
exprès. Littéralement, au moment de la sortie de ce livre sur l'exil, j'avais les jambes engourdies. Comme ce n'était pas une maladie douloureuse, néanmoins très impressionnante, je ne pouvais pas fonctionner normalement, cela m'a beaucoup donné à réfléchir. Même si, comme l'a montré Susan Sontag, l'on a parfois tendance abusive à lire les maladies comme des métaphores, à psychologiser tous les problèmes médicaux, les neurologues qui ont travaillé sur mon cas m'ont dit que ça ne pouvait pas être psychosomatique, du fait que la paralysie montait progressivement comme ça. Néanmoins, moi je l'ai vécue comme une métaphore, comme un message que m'adressait mon corps et qui était de l'ordre d'une «congélation" de mes racines. Et toutes les images qui me venaient en tête à ce moment-là étaient les images du Canada et de mon enfance, dans la neige, ou à la patinoire, les pieds gelés par le froid pour être restée longtemps dehors à faire du patin. C'est à partir de ce moment-là que je me suis dit : oui, mes racines sont gelées, il faut que je ranime en moi la langue anglaise. Ce n'est pas que, dès ma guérison, je me sois mise à écrire en anglais, mais au sortir de cette maladie Trois fois septembre, que j'ai écrit en 87-88, c'est le roman de la transition. C'est-à-dire que l'on est déjà aux États-Unis, mais avec une fille qui traduit pour sa mère ce qui est écrit en anglais, etc. Donc je l'ai écrit en français, mais c'est déjà un français pervers, si j'ose dire, parce que tout est censé être traduit au fur à mesure. Je disais déjà que je vais vers la langue anglaise, n'est-ce pas? Ce texte est fictivement«en anglais» même si on le lit en français.

MK.Y. : Ce qui est passionnant dans ce roman qui nous fait découvrir - à travers ses divers écrits, correspondances et journal intime -, la trajectoire tragique d'une jeune fille américaine qui finit par se suicider c'est, comment dirais-je, une sorte de jeu de renversements : tout d'abord, il y a en effet comme vous le dites que le lecteur se voit obligé de s'en remettre au travail de traduction qu'accomplit pour sa mère, Solange, une jeune fille venue de la France et liée d'amitié avec Selena. C'est-à-dire, l'histoire que le lecteur lit en français est censée avoir été originellement écrite en anglais par la jeune fille américaine tragiquement disparue. Deuxièmement, il y a un renversement de position : compte tenu du fait que l'auteur est un écrivain d'origine anglophone mais installé en France, on ne peut qu'être intrigué de suivre l'histoire de Selena par l'intermédiaire de deux Françaises, une jeune fille et sa mère qui après la mort de l'homme, père de l'une et mari de l'autre se sont transplantées aux ÉtatsUnis. Qui plus est, ce que vous venez de dire me frappe encore plus, du fait que dans le roman, vous faites mourir la jeune Américaine qui rêvait de devenir comme l'arbre plongeant ses racines solides dans le sol.

N.H. : Elle se suicide en se pendant à un arbre, qui plus est. 
MK.Y.: Qui est précisément un érable.

N.H. : Oui, un érable (rire)... Je me souviens que pendant tout le temps de la rédaction de ce roman, j'étais hantée par les images de l'érable. Je l'ai écrit entièrement sur du papier rouge. C'est un roman sur la maladie mentale, mais aussi c'est un roman pour lequel j'ai mal contrôlé la distance entre moi et Selena, en ce sens que cela m'a vraiment plongée là où je n'avais pas envie d'aller. II a fallu laisser passer un an, entamer une nouvelle grossesse, entamer l'écriture du Journal de la création. Et dans la foulée, j'ai retravaillé Trois fois septembre pour le rendre recevable, parce que la première version était un objet asocial, pas tout à fait littéraire, trop violemment «intérieur». Mais c'était un tournant important pour moi, ce livre. Ce n'est pas un de mes meilleurs, j'en suis consciente; mais personnellement, je sais le rôle qu'il a joué dans ma trajectoire.

MK.Y. : Je pense que la lecture du roman permet de percevoir cette importance que vous lui accordez. Un autre point qui retient mon attention dans ce roman, c'est la présence de la mère nommée Renée, installée dans une position de lectrice-soutien, pour qui et grâce à qui sa fille Solange traduit les témoignages de Selena, parfois douloureux. Un passage du roman nous montre Solange en train d'ouvrir une lettre de son amie disparue et par là, obligée d'avouer à sa mère :«je ne t'ai pas tout raconté». Une petite tension se crée alors entre elle et sa mère. Cela m'amène à vous poser la question suivante : est-ce que pour vous, la transplantation linguistique, même si à présent vous avez fait retour à votre langue maternelle, est une chose sans quoi votre venue à l'écriture aurait été irréalisable?

N.H. : Tout simplement oui. Je ne sais pas, c'est impossible de dire ce que je serais devenue. Dans la meilleure des hypothèses, j'aurais fait partie de ce monde que j'évoque dans La Virevolte et Dolce Agonia, c'està-dire le milieu universitaire de la côte est américaine, probablement j'aurais été professeur, qui sait. J'avais un très grand désir d'écrire. Je me souviens que je suis arrivée en France en disant à qui voulait l'entendre que c'était mon projet dans la vie d'être écrivain. J'avais suivi des ateliers d'écriture, aussi bien au lycée dans le New Hampshire qu'à New York, à l'université avec E.L. Doctorow (l'auteur de Ragtime). Mais il m'a fallu et le détour par la langue française et aussi, curieusement, le détour par la théorie, même si maintenant j'ai l'impression d'avoir perdu beaucoup de temps dans des séminaires abscons. Pour la jeune femme psychologiquement assez fragile et un peu «paumée" que j'étais à l'époque (entre mes dix-sept et vingt ans), c'était très rassurant de tomber dans un monde où tous avaient des certitudes, psychanalytique, structuraliste, 
marxiste etc. Tout cela était très euphorisant. Et les idées elles-mêmes étaient excitantes, les gens étaient très passionnés et avaient énormément de convictions et d'éloquence. Et je me suis dit que tous mes problèmes personnels - littéraro-personnels - étaient des problèmes petit-bourgeois, nombrilistes qui n'avaient pas lieu d'être et qu'au lieu de s'en préoccuper, il fallait faire la Révolution! J'ai à peu près délaissé mes ambitions littéraires pendant cinq ans, à quelques exceptions près, car ce que j'ai écrit dans Sorcières était malgré tout littéraire, mais avec l'idée que cela s'inscrivait dans la lutte des femmes. Finalement, je crois que cela m'a donné des forces, ces années-là où j'ai ingurgité énormément de livres et d'idées, je me suis sentie pour la première fois de ma vie faire partie d'un groupe. Ce n'est quand même pas pour rien que les gens créent ces idéologies, ça pallie beaucoup l'angoisse personnelle. Maintenant je me sens plus proche de celle que j'avais été avant d'arriver en France que de cette intello parisienne de mes premières années à Paris. Le détour par la langue française a été fondamental, et pas seulement pour la raison que vous avez citée. II y avait aussi que c'était difficile à déchiffrer pour mes parents ... et encore, inégalement, parce que ma mère parle bien le français, elle est psychologue et reçoit des patients en français. Mon père n'est pas francophone, mais il est capable de se servir d'un dictionnaire. Donc la barrière que représentait le détour par la langue française était une barrière poreuse, mais utile pour moi. Je crois que c'est vrai pour tous les écrivains. Boris Cyrulink dans son livre Un Merveilleux malheur discute du fait que bon nombre d'écrivains ayant subi un traumatisme dans l'enfance ont pu développer de la résilience et des forces qu'ils n'auraient pas développées autrement. C'est vrai, mais l'absence des parents libère aussi des paroles qui auraient été censurées par leur regard intériorisé. C'est quand même normal de vouloir ne pas blesser ses proches! Donc le fait de mettre un océan et une langue entre eux et moi m'a permis de dire des choses que je n'aurais pas osé dire.

MK.Y. : Ce qui semble transparaître dans vos premiers écrits, c'est l'idée que l'écriture est presque quelque chose de coupable.

N.H. : Non, ce que j'avais à dire était parfois des choses blessantes pour eux, mais l'écriture en elle-même n'est pas coupable, seulement transgressive par rapport à, pour dire par euphémisme, la courtoisie. C'était impensable, par exemple, de dire à nos parents que nous avions beaucoup souffert de leur arrangement familial. Eux estimaient que c'était la meilleure solution, qu'ils avaient pas mal rafistolé les choses, et qu'on avait grandi dans des conditions plutôt heureuses, ce qui n'est pas faux.... II y avait une sorte de déni du traumatisme qui était gigantesque. C'était comme une bombe plantée dans notre cœur. On avait l'énergie de conte- 
nir cette bombe en nous. Entre autres, les romans, pour moi, étaient la possibilité de laisser s'échapper un peu de cette rage contre la violence qui m'avait été faite. L'empreinte de l'ange n'est que ça; c'est moi Emil, moi que l'on a jeté du train à l'âge de six ans, avec les trois adultes qui décident de tout, sans tenir compte des besoins de l'enfant, ni de ses questions, ni de son identité, etc. Je peux décrire la dynamique du livre ainsi maintenant, mais je ne le savais pas du tout en l'écrivant. Ce livre-là n'aurait pas existé sans cette structure familiale très particulière qui était la nôtre. Bien sûr le roman ne peut se réduire à ça, je l'espère! Je voulais revenir un instant à l'image de l'arbre. II y en a une autre que j'utilise. Quand on voit un arbre qui pousse entouré d'une grille en fer forgé... il a réussi à se développer en se tordant; finalement il existe. Je me vois beaucoup comme ça, comme si j'avais un grillage de fer à travers le cœur. Pourquoi ce détour par la langue française? C'est ça la torsion. Si j'avais pu grandir tout droit, normalement, comme tous les autres arbres, je l'aurais fait. Mais à cause de ce grillage planté dans mon cœur, j'ai dû pousser de façon tordue, mais j'ai tout de même poussé.

MK.Y. : La courtoisie pour les proches dont vous parlez et qui est une très jolie formule était sans doute déterminante pour les contenus. Mais est-ce qu'il n'y aurait pas quelque chose qui touche aussi à la forme et qui vous aurait également imposé la nécessité du détour par une langue étrangère? C'est ce qui me semble ressortir à la lecture de Lettres parisiennes: Histoires d'exil où vous faites état de votre difficulté à supporter la sonorité, la musique de la langue anglaise.

N.H. : Il faut tenir compte du fait que j'ai écrit cela avant mon retour à la langue anglaise, justement. Je peux souscrire encore à certaines choses énoncées, par exemple, dans Désirs et réalités notamment à propos du caractère «agressif» de la langue anglaise que j'entends dans les rues de Paris. Je la sens comme une chose incongrue, mal polie, mais l'américain me fait plus cet effet que l'anglais de l'Angleterre. Pour autant, je ne pense pas que la langue française a des qualités intrinsèques qui la rendent, par exemple, plus fluide, plus cartésienne, plus jolie, plus claire, que sais-je encore. J'aurais pu choisir n'importe quelle langue étrangère. C'est pourquoi je n'ai rien en commun avec les champions de la francophonie. II se trouve que c'était la langue française, non pas à cause de mes origines canadiennes, mais parce qu'au lycée, j'ai eu un très bon professeur de français d'une part et, d'autre part, mon université new-yorkaise avait une antenne à Paris. Mais si ça avait été l'italien, l'effet intérieur, psychologique aurait été identique. L'important était que l'anglais était grevé de deux façons symétriques et inverses : d'une part, c'était la langue de l'université et des études, donc des clichés universitaires et des phrases 
toutes faites; qui représentent un immense handicap pour quelqu'un qui veut écrire. La langue française, en revanche, était «vierge». Dans la langue française, je n'avais pas ces habitudes qui paralysent tant d'écrivains français; (j'ai l'impression qu'aux États-Unis le système scolaire est un peu moins rigide ).... J'avais moins de «surmoi» en français. N'ayant pas étudié le canon français, je n'avais pas installé sur un piédestal intérieur Rimbaud, Flaubert, Baudelaire, Balzac, etc. Je vois bien comment on enseigne tout cela à ma fille; je vois bien à quel point pour elle il est inimaginable de pouvoir s'exprimer par la littérature, de dire quelque chose de frais, d'authentique, tellement les formes sont là, intimidantes. Voilà pour le côté «surmoi». Et puis il y a le côté "ça»... Je me permets d'utiliser ce schéma freudien parce que vous êtes psychanalyste.... Donc "ce n'est pas pour rien", comme disent les psychanalystes, que j'ai fait mon mémoire de maîtrise sur les gros mots, les mots blasphématoires, les interdictions linguistiques, les tabous linguistiques Dire et interdire : éléments de jurologie. J'aurais été incapable de faire ce travail en anglais, parce que la force émotive de ces mots était efficace dans ma langue maternelle, alors qu'en français, mon hémisphère gauche est concerné mais pas le droit. Ce n'est sûrement pas aussi simple que cela, bien sûr, mais ça facilite la conceptualisation de la chose. Je ne peux pas être atteinte en profondeur, par exemple, par une injure française, ni sentir vraiment la «saleté» d'un mot français quel qu'il soit. En anglais, j'aurais eu du mal à avoir une distance nécessaire pour mener à bien un travail universitaire sur les interdictions linguistiques.

MK.Y. : En effet, ce que vous dites là correspond à ce que montrent les études psychanalytiques sur les sujets bilingues ou polylingues, en particulier, des personnes fermement décidées à s'exprimer uniquement dans leur langue d'adoption. Au risque d'une simplification, on peut dire que la langue maternelle est chargée d'un poids affectif et d'une série de traces sensorielles et corporelles, ainsi que des fantasmes les plus archaïques issus des premières étapes de la vie de chacun.

N.H. : C'est la raison pour laquelle je voulais revenir à l'anglais. Je me suis dit : je peux continuer d'écrire des livres en français comme Les Variations Goldberg. Mais si je coupe tout contact avec ce niveau archaïque, mes romans resteront «cérébraux». Pendant des années, la langue française agissait comme un bouclier pour me protéger des émotions liées à mon enfance. Mais, une fois que j'avais acquis un certain métier, une certaine habitude de l'écriture et de la publication, je pouvais prendre le risque d'ouvrir ces vannes. Je savais que je n'écrirais rien de valable si je restais uniquement dans la langue française. En même temps, on pourrait dire presque le contraire de ce que je viens de dire : on 
a commencé par dire que je pouvais exprimer des choses tabous dans la langue étrangère et c'est vrai que parfois je peux dire des choses beaucoup plus violentes, plus coupantes, plus sanglantes en français parce que les mots ne portent pas la même charge pour moi que pour un francophone.

MK.Y. : Il est vrai que les travaux de recherche que j'ai signalés tout à l'heure semblent souligner dans un premier temps comment l'usage d'une seconde langue remplit une fonction défensive à l'égard des vécus infantiles, sources d'une angoisse intolérable. Néanmoins, ils attirent aussi l'attention sur la manière dont le détour par une seconde langue contribue à la réorganisation des conflits identitaires; souvent l'acquisition et l'utilisation d'une langue étrangère peuvent jouer le rôle de voie d'accès à une contrée psychique identitaire qui serait restée autrement inatteignable.

N.H. : C'est évidemment le cas d'Agota Kristof qui est un écrivain d'origine hongroise, installé en Suisse et qui n'écrit qu'en français. Ses livres, très frappants tant par leur violence que par leur dépouillement, sont écrits dans un français qui n'est marqué par aucun signe d'appartenance, ni sociale ni régionale; ils sont très noirs, remplis des images d'une violence insoutenable - guerres, destructions, meurtres, suicides - difficile à situer historiquement ou géographiquement. Je sais qu'elle n'aurait pas pu écrire tout cela dans sa langue maternelle et qu'elle est protégée de sa propre violence par l'écran de la langue étrangère.

MK.Y. : On peut aussi relever une figure plus classique, par exemple Samuel Beckett. II est connu que, vers la fin des année 30, cet écrivain irlandais a entrepris de composer ses livres directement en français. La transplantation linguistique et géographique lui aurait permis de dépasser l'inhibition à écrire dont il avait souffert jusque là; on peut dire qu'une grande majorité de son œuvre qui a marqué notre temps a pris corps dans la langue d'emprunt qu'était pour lui le français. Et puis, après la mort de sa mère, il a fini par revenir à l'anglais. Si j'évoque l'exemple de Beckett, c'est pour la question de la traduction. Un peu comme vous, Beckett s'est chargé lui-même de la traduction de ses livres d'une langue dans l'autre. Or, selon de nombreux critiques littéraires, Beckett mettait, dans le passage de la traduction, un grand soin à ne laisser transparaître dans la langue d'arrivée aucun signe, aucune ombre de la langue du texte original. En sorte que les deux langues ne conservaient aucun souvenir l'une de l'autre et qu'il n'y avait aucune communication entre les deux.

N.H. : C'est effectivement mon cas aussi : je traduis tous mes romans dans un sens ou dans l'autre. 
MK.Y. : Est-ce que votre travail de traduction opère une telle coupure entre les deux versions, comme Beckett qui remaniait complètement son texte original pour la traduction?

N.H. : Ce n'est pas vrai que ça soit remanié. Vous pouvez lire L'Innommable. On ne peut jamais douter qu'il y ait une autre version, parce que c'est tellement idiomatique, c'est tellement évidemment cette langue-là. Mais si vous comparez les deux versions terme à terme, vous verrez qu'elles sont identiques, phrase pour phrase. Beckett n'est pas Julien Green, ni Romain Gary. C'est une question à laquelle je me suis beaucoup intéressée. En traduisant La Danse de Gengis Cohn du français en anglais, Gary supprime des chapitres entiers, il en ajoute d'autres, il adapte les blagues à son public; en somme, c'est une réécriture. Julien Green prenait le même point de départ pour son autobiographie et puis il se rend compte qu'il n'est pas du tout la même personne en français qu'en anglais, et qu'il s'en va vers un tout autre livre! En ce qui concerne les techniques de la traduction, je suis plus proche de Beckett, c'est-à-dire qu'il y a deux versions de tous mes romans, sauf le Trois septembre qui n'existe pas en anglais (je l'ai traduit, mais il n'a jamais été publié) et j'espère que les traductions dans les deux langues peuvent être lues dans la langue des lecteurs. En tout cas pour moi, c'est important qu'il ne manque pas une page ou un paragraphe et que l'éditeur américain approuve ce que l'éditeur français approuve et inversement. Je tiens à ce que les deux versions soient identiques dans la mesure du possible. Limbes, c'est un livre qui rend hommage à Beckett. Le succès de Instruments des ténèbres auprès du grand public français m'a troublée et m'a fait me demander si je voulais être un «écrivain français". Soudain j'ai eu une sorte de chute identitaire.... Là je me suis dit que c'était vraiment la problématique de Beckett, à savoir : à partir du moment où je peux être Irlandais et Français, qui sont mes personnages? Et où se passent mes histoires? Qui sont mes lecteurs?, etc. Beckett a décidé de s'abstraire complètement de ces questions, enlevant le corps - vous avez parlé tout à l'heure de prendre corps -, retranchant donc le corps et ne s'occupant que de l'intérieur de la tête, qui, lui, est grosso modo identique dans le monde entier, ou tout du moins dans le monde occidental moderne.... Ses personnages n'ont pas de noms vraisemblables, ni de famille, ni d'ancêtres, ni de métier, ni de nationalité, c'est-à-dire que ce sont de purs esprits, malheureux, faméliques, incarnant une sorte de misère d'être. Beckett est génial parce que, ce faisant, il écrit des pièces universelles qui, tout en parlant du non-sens de l'être, ont un sens partout. Si j'écris l'histoire de jumeaux berrichons sous l'Ancien Régime en 
France, j'écris tout à fait autre chose. Qui s'identifie à ça? Tout Berrichon, d'abord et puis les jeunes. Ce n'est pas pour rien que Instruments des ténèbres a eu le prix du Goncourt des lycéens. Cette question d'identification est très intéressante; on peut aimer un roman parce qu'il vous raconte ce que vous connaissez, ou parce qu'il vous entraîne loin, vous fait rêver à un monde exotique. Limbes est un cri de détresse, un cri de perte identitaire, c'est ça les limbes; je ne voulais pas être un écrivain français; je ne suis pas - je ne peux pas être - un écrivain canadien, je n'ai jamais écrit un mot au Canada. C'est là que j'ai compris que je faisais partie d'un groupe d'écrivains divisés. L'empreinte de l'ange est aussi un roman qui reflète cette crise-là; c'est un roman situé dans un Paris qui n'est pas du tout paradisiaque, un Paris dans la tourmente de la guerre d'Algérie, avec la question posée à chaque page :«qui s'identifie à qui»? Est-ce qu'une Allemande peut s'identifier aux Algériens, un Juif à une Allemande, vous les lecteurs à un petit enfant franco-allemand, à ces petits enfants juifs, à ces militants algériens, etc? Et jusqu'à quel point? D'une certaine façon, la solution de Beckett est la seule qui soit élégante : on annule le monde. Mais, personnellement, j'ai besoin des histoires, je ne peux pas écrire d'histoires des personnages qui ne sont pas vivants, il faut qu'ils aient un corps, des parents, une maison, etc.

MK.Y. : La question de l'identité dans le monde littéraire, cette possibilité latente d'ouverture identitaire proposée aux lecteurs, peuvent trouver une solution dans deux paris extrêmes : l'un est représenté par Beckett qui ôte à ses personnages tout ancrage matériel, concret, réel pour les élever de la sorte à une dimension universelle, l'autre inauguré par Montaigne qui vise à atteindre l'universel, en approchant de plus près à ce qu'il y a de plus singulier.

N.H. : Absolument. Dans un essai intitulé Le Déclin de l'identité? je parle de trois types d'écrivains, les uns étant enracinés, comme Montaigne Toni Morisson, Giono - qui arpentent leur petit monde et parviennent à creuser si profond, si loin que tout le monde est capable de s'y reconnaître. Ces écrivains n'ont pas besoin de parcourir le monde entier. Et puis, il existe des écrivains comme Romain Gary que je qualifie "d'éparpillés" et de qui je me sens proche. Gary avait au départ une identité tellement multiple, tellement fragmentée, qu'il ne pouvait qu'essayer de mettre cela au service de son don de romancier et de s'en servir pour créer des personnages incroyablement divers. Un autre type d'écrivains est ce que j'appelle écrivain «divisé», comme Leïla Sebbar, Vassili Alexakis, Agota Kristof, etc, qui ont vécu une partie de leur vie ici et une autre là-bas. Montaigne, c'est bien. Mais il se trouve que pour moi, les 
racines ne vont pas bien loin. Ni mes racines en France, ni mes racines au Canada, ni mes racines aux États-Unis ne me permettent de me dire que ça c'est mon monde, mon petit arpent de terre, qui contient l'univers que je veux explorer. Je suis faite de trop de choses différentes.

MK.Y. : Est-ce que l'on se sentirait poussé à écrire, lorsque l'on croit posséder complètement ne serait-ce qu'un petit bout de terre? Plutôt qu'un sentiment de possession inébranlable, ne serait-ce pas un sentiment d'incertitude, d'écart irréductible qui met en mouvement l'écriture? Là, je songe à la fin de votre livre sur l'exil où vous avancez l'idée que peut-être le mot exil n'exprime rien d'autre qu'un sentiment de division interne, de distance intérieure qui était vitale, nécessaire à l'écriture.

N.H. : Oui, une distance que, par le mot ou la situation exil, l'on a réifiée. Je ne sais pas si les écrivains enracinés comme Victor Hugo partaient de ce même sentiment de division intérieure qui m'est si constitutif. Je ne crois pas que l'on puisse généraliser cela. Prenons l'exemple de Toni Morrison : I'histoire des Noirs américains, l'époque de la fin de l'esclavage, il y a eu des horreurs, il y a eu des comportements moraux très complexes, tout cela est d'une richesse infinie. C'est un univers qu'elle peut explorer toute sa vie; Morisson n'a pas besoin d'aller voir l'Espagne à l'époque de l'Inquisition. Mais quelqu'un comme Romain Gary, qui est né en Russie, qui a vécu en Pologne, avant d'arriver en France et de faire la guerre en Angleterre, qui a travaillé comme diplomate à Sofia, en Amérique du Sud et en Californie...il a trop vu pour parler à partir de ses «racines" en tant qu'écrivain.

MK.Y. : Selon vous, il existerait donc une catégorie d'écrivains chez qui l'écriture repose sur un sentiment de possession de leurs racines.

N.H. : Oui, mais encore une fois ce n'est pas une question que l'on se pose quand on s'assoit à sa table de travail. C'est seulement après-coup que l'on peut dire que c'est ce type de rapport à l'identité qui est donné. Évidemment, on devient écrivain en problématisant ce qui est donné.

MK.Y. : Pour rebondir sur votre expression de problématiser ce qui est donné comme racines ou identité, je serais tentée de soutenir l'idée suivante : ce qui transparaît avec la plus grande force et la plus dramatique intensité chez les écrivains divisés, c'est la question de l'identité surgissant davantage comme une question posée que comme une réponse donnée. Peut-être les écrivains en exil, au sens propre ou au sens figuré, sont mieux placés pour être écrivains.

N.H. : Tout à fait. C'est la question que j'explore dans Nord perdu que l'on tient pour un livre désespéré, alors que c'est un livre plus serein que 
l'époque de Limbes. Je dis là que les expatriés connaissent un certain nombre de vérités concernant la condition humaine, notamment le caractère arbitraire de l'identité. À force de voyager, de se laisser transformer par l'apprentissage d'une langue étrangère, on voit à quel point ce qu'on prenait pour «soi" est une construction faite de beaucoup de hasards. La question de la langue, notamment, est vertigineuse.

MK.Y. : Votre expérience de traduction de vos propres livres rejaillit-elle également sur vos textes d'origine?

N.H. : Oui. C'est pourquoi, depuis Cantique des plaines, je fais toujours une version originale, ensuite la traduction qui m'aide à réviser l'original. Donc quand je dis que je tiens que les deux soient identiques, c'est vrai, mais ça ne veut pas dire que je tiens à ce que le français soit le reflet parfait de l'anglais ou inversement, parce que justement quand je vois une phrase mal bâtie dans la version originelle, grâce à la traduction, je peux l'améliorer. Pour La Virevolte il y a eu des aller-retour assez chaotiques entre les deux langues.

MK.Y. : Quelle figure métaphorique pourriez-vous prendre pour caractériser vos aller-retour entre les deux langues dans votre travail d'écriture et de traduction?

N.H. : Je ne sais pas. Je trouve surtout que c'est un travail long et fatigant. Sur le plan psychanalytique, je dirais que c'était comme si je faisais l'aller-retour entre mon père et ma mère, avec le sentiment qu'au fond, si seulement ils voulaient bien s'écouter, ils verraient qu'ils disent la même chose. Autant je trouve ce travail fastidieux, autant quand j'en suis à la deuxième version, je constate que c'est le même livre, j'éprouve une immense satisfaction. Quand j'entends la musique de la langue d'arrivée, quand ça commence à produire l'effet recherché, c'est gratifiant, alors que le chemin qui y conduit est sans aucun intérêt.

MK.Y. : Même si maintenant vous écrivez directement en anglais, on a le sentiment que le travail de traduction tient lieu de l'expérience du détour par la langue française.

N.H. : Je ne dirais pas cela. Je ne sais pas si j'ai encore besoin de l'illusion d'être à l'abri d'un regard familial, mais en tout état de cause je me suis rendu compte que ma famille s'en moquait pas mal et que j'étais donc libre. Et puis c'est surtout que... j'ai l'impression de dire la VÉRITÉ quand j'écris. Pas de façon détournée, masquée. J'ai l'impression de partir de très loin, que ce soit en français ou en anglais, que l'histoire de mon roman se passe dans le Marais ou en Afrique du Sud, j'ai l'impression que c'est très loin, de toute façon, d'être dans le coeur ou la tête de quelqu'un 
d'autre. Et ça me suffit comme transport; c'est très «transportant».

MK.Y. : II me semble que par ce transport, vous opérez un retournement en votre faveur de quelque chose qui a été blessant au départ, à savoir ce sentiment de division et de blessure.

N.H. : C'est ce que j'ai essayé de dire à propos de Romain Gary qui disait plaisamment à propos du caméléon qu'il devient rouge quand on le pose sur du rouge, qu'il devient vert sur du vert, mais quand on le pose sur un plaid écossais, il devient fou (rire). Et Gary, malgré le«plaid écossais» qu'était son enfance, disait avoir été protégé contre la folie par la création littéraire. Néanmoins cela ne l'a pas protégé jusqu'au bout, puisqu'il a fini par se suicider.

MK.Y. : Ce qui rend puissants ces écrivains qui exaltent l'expérience de division et célèbrent la douleur d'exil géographique ou interne, ne seraitce pas le fait que leur création littéraire date de la blessure liée à l'origine qui dépasse même telle situation familiale complexe ou tel parcours tortueux? Pour le coup, on pourrait se demander si toute création littéraire n'impliquerait pas une part de la quête de l'origine.

N.H. : Là je ne vous suis pas du tout. Pour moi, l'idée de quête de l'origine est dépourvue de résonance. Je n'ai pas l'impression du tout de la chercher, je suis tout sauf nostalgique, je suis entièrement tournée vers les projets. Si vous dites que les projets sont formés ou déformés par la dynamique de ce qui vient de l'enfance, je veux bien, mais je ne suis pas poussée à fouiller dans mon passé, me demandant où est mon origine.

MK.Y. : Bien entendu, je ne pense pas que l'on puisse considérer l'origine simplement comme quelque chose qui nous tire en arrière. L'origine est aussi comme un point lointain qui scintille au loin et devant soi. À ce propos, j'aimerais bien évoquer un extrait d'un poème coréen qui s'appelle Sur la route. Par ailleurs, je dois préciser que le mot coréen signifiant«la route» ou«le chemin» a une résonance infinie comme un point de fuite en peinture. «Je suis sur la route à la recherche de quelque chose que j'ai perdu... Je suis sur la route pour ne pas savoir ce que j'ai perdu". L'origine ou l'identité, c'est justement ce que l'on croit avoir perdu sans pour autant l'avoir jamais complètement possédé.

N.H. : J'ai une image plus constructiviste de l'identité. Je ne pense pas avoir eu une identité forte et fixe qui a été fracassée. Je pense que je suis le produit de tout ce que j'ai vécu et appris, des gens que j'ai rencontrés et des interactions amicales, amoureuses et conflictuelles que j'ai pu avoir. Je pense aussi comme ce que disait Marina Tsveta'va : tout ce qui vous empêche d'écrire, c'est là votre véritable biographie créatrice. Autrement dit, c'est encore l'image du grillage planté dans le cœur qui 
revient. En exprimant ce que je peux exprimer grâce à mes propres torsions et blessures, je touche à quelque chose de plus universel que les autres vivent dans leur rêves. C'est ça l'identification littéraire au sens le plus noble du terme. Quand on arrive à entrer dans la vie des gens, comme un esprit humain en général, non pas comme une Allemande s'identifie à une Allemande, un Juif à un Juif, etc.

MK.Y. : J'aimerais aborder quelque chose qui me semble constamment présent dans vos romans : il s'agit de la présence d'une relation du double qui apparaît tantôt sous la forme d'une relation entre deux jumeaux ou de deux adolescentes ou encore de deux amoureux étrangers l'un à l'autre, qui sont issus des camps ennemis : l'un représente l'enracinement, tandis que l'autre figure l'errance. Ce qui m'a frappée, c'est que c'est toujours celui ou celle qui reste attaché à l'origine, comme cet arbre ancré dans le sol, finit par se détruire, - on serait presque tenté de dire : se sacrifier - ; c'est le cas de Selena dans Trois fois septembre, comme c'est le cas du frère jumeau de Barbe dans Instruments des ténèbres et puis d'une certaine manière, on peut en dire autant pour Raphaël dans L'empreinte de l'ange.

N.H. : Cela, je peux l'entendre, mais je ne peux pas analyser mes propres romans. Je ne peux pas être à la fois dedans et dehors. II faut que je laisse cette tâche aux commentateurs. C'est pourquoi je ne suis pas allée au colloque consacré à mes romans. Je trouve qu'il serait malsain d'acquérir un savoir sur la manière dont fonctionnent mes romans.

MK.Y. : À lire vos romans, on peut avoir le sentiment d'assister à une célébration du voyage, du départ et de l'errance qui sont, me semble-t-il, aussi bien une manière de se séparer de l'origine que de la retrouver. Comme disait plaisamment un savant russe qui, paraît-il, parlait une dizaine de langues : on ne parle pas une dizaine de langues, on parle sa langue maternelle en autant de langues. Peut-être une dernière question: vous dites dans Nord perdu que lorsque l'on est étranger, on retrouve la position de l'infans.

N.H. : Mais c'est l'étranger qui ne parle pas la langue du pays, pas comme moi en France. Quand je parle de la détresse de l'étranger, c'est de celle de l'enfant sans parole qu'il est question. On grandit à travers l'apprentissage de la langue. On parle d'abord comme un enfant, faisant d'énormes fautes de grammaire et de syntaxe; bien sûr on n'apprend pas de la même manière qu'un enfant, mais comme une sorte de cours accéléré, on arrive à l'âge adulte en cinq ans.

MK.Y. : N'y aurait-il pas quelques restes de l'infans que l'on retrouve dans la position de l'étranger? 
N.H. : Non, je ne le pense pas. Au contraire, comme on le disait au début, on ne peut avoir une deuxième enfance dans une langue étrangère, avec ce que l'enfance implique de vulnérable, de subi. On a déjà fait tout cela dans son enfance, il n'y a pas deux enfances dans le sens profond du terme. On se sent infantilisé, handicapé, bête, réduit à des choses simples, frustré. Quand on a dix ans, on écoute les adultes sans les comprendre, et puis soudain à quinze ans, tout ce que disent les adultes devient transparent. Cette expérience-là peut se retrouver dans l'apprentissage de la langue étrangère, mais la petite enfance, non, je ne crois pas que l'on puisse la revivre.

MK.Y. : Après tant d'années passées en France, vous sentez-vous toujours étrangère dans ce pays?

N.H. : Je suis étrangère partout, absolument partout, mais je me débrouille mieux que la plupart d'simpatriés". Je me sens complètement à l'aise, que ce soit ici, dans le Berry, au Canada ou aux États-Unis. Je maîtrise les codes de communication linguistiques et culturels, je me sens de plain-pied, mais je me sens étrangère.

MK.Y : Pensez-vous que ce sentiment d'être étrangère en permanence continue à intervenir dans votre écriture?

N.H. : Là aussi c'est aux autres de le dire. Je n'aime pas du tout qualifier mon écriture. C'est pour ça que je me disais tout à l'heure incapable de parler de mon style. C'est très mauvais de mélanger les deux niveaux de discours. Ce n'est pas à nous de savoir.

MK.Y. : Ce qui m'amène à évoquer cette question est lié à ce que vous dites au sujet de votre langue maternelle. D'une part, vous relevez cette charge charnelle et émotionnelle qui grève la langue maternelle, tout en la rendant vivante au point d'en entraver l'usage libre. Et d'autre part, vous dites qu'une partie de cette langue se trouve pour ainsi dire morte, fossilisée, si bien qu'un travail de déconstruction ou de déstructuration s'impose pour rendre possible votre écriture. Comme dans le cas de Beckett, vous avez opéré ce travail de déstructuration d'abord sur - ou grâce à - une langue d'adoption qu'est le français.

N.H. : Je dis souvent, - par ailleurs je ne suis pas la seule à le dire -, qu'il faut traiter de la langue maternelle comme une langue étrangère. Il y a des écrivains qui arrivent à le faire dans leur langue maternelle, sans le détour par une langue étrangère. Mais pas beaucoup d'universitaires. II arrive qu'un écrivain parvienne à très bien enseigner, comme André Brink ou Michael Ondaatje. Par contre lorsqu'un universitaire, quelqu'un qui a fait une thèse, se propose d'écrire, je pense que c'est très difficile. J'en 
connais très peu. Sylvie Germain qui a fait une thèse de philosophie est un très bon écrivain, mais c'est un cas exceptionnel.

MK.Y. : À dire que la langue maternelle est à la fois trop vivante par son côté charnel et trop morte par usure rigide.

N.H. : Oui, c'est pour ça que je parlais tout à l'heure du côté«ça» et du côté «surmoi». L'écriture a besoin d'un maximum de ça et d'un minimum de surmoi, et l'écriture universitaire a besoin d'un minimum de ça et d'un maximum de surmoi. Quand, en 1989, je suis revenue à la langue anglaise pour écrire Cantique des plaines, je ne m'étais pour ainsi dire pas servie de l'anglais depuis dix ans. Je l'enseignais, j'avais quelques amis anglophones, mais je le lisais très peu dans ma langue maternelle. Quand je l'ai récupérée, elle était pour moi presque plus étrangère que la langue française. Le français était devenu la langue du quotidien, la langue du supermarché, des impôts, de la banque; bref, une langue fonctionnelle, comparée à ce qu'elle était au début pour moi par sa musicalité, cette sorte de beauté phonétique que je découvrais au fur à mesure. Au début, j'entendais le français, comme un francophone ne pouvait l'entendre. Eh bien, grâce à la longue désaffection, l'anglais m'était devenu presque aussi neuf, aussi frais; cela se sent dans le livre Plainsong; si on le lit à voix haute, on sent la jubilation de mes retrouvailles avec cette langue, je me roule dedans, comme un cochon dans la boue. J'adore!

MK.Y. : Notre entretien touche à sa fin. Et j'aimerais vous demander ce que vous pensez de mon intention de placer notre dialogue sous le titre de «l'Épreuve de l'étranger», titre qui me semble correspondre aux expériences sous-jacentes à notre dialogue : exil, littérature, psychanalyse. Est-ce que pour vous si je disais que la littérature est une forme de l'épreuve de l'étranger, cela pourrait-il vous convenir?

N.H. : Je ne sais pas. C'est un peu abstrait pour moi.

MK.Y. : Cette idée m'a été suggérée par un des personnages de vos romans, la romancière, héroïne créatrice de Instruments des ténèbres. Cette romancière a comme compagnon de création, comme allié, le diable, le daîmon qui représente une figure diabolique, un être qui divise, qui jette à travers. C'est là aussi la métaphore du voyage, de l'errance qui surgit. Dans ce sens là, ne pourrait-on pas parler de l'expérience littéraire comme une forme de l'épreuve de l'étranger?

N.H. : Mais entendez-vous par le mot«épreuve» un examen ou une souffrance?

MK.Y. : Ah oui! eh bien, plutôt au sens d'une mise à l'épreuve, donc au sens de l'éprouver qui ne peut exclure le sens d'examen. II est vrai que 
dans Lettres parisiennes, vous dites que vous avez tellement écrit en anglais pour passer des examens, vous avez l'impression de passer un examen, à chaque fois que vous écrivez en anglais

N.H. : Cela m'arrive encore d'avoir le sentiment de subir un examen, comme par exemple dans un entretien en langue anglaise. L'expression de l'épreuve de l'étranger ne sonne juste dans mon cas, - dans la mesure où je peux juger de mon propre cas - que dans cette histoire d'autotraduction. Là je me soumets à l'épreuve de l'étranger, c'est-à-dire : est-ce que cela raconte encore la même histoire dans l'autre langue, est-ce que cela suscite les mêmes émotions, est-ce que cela parle des mêmes personnages?

MK.Y. : C'est-à-dire dans la traduction de l'anglais en français?

N.H. : Ou l'inverse, comme c'est le cas pour L'Empreinte de l'ange ou Prodige

MK.Y. : Là, peut-être je pourrais rapporter un témoignage de mes expériences de psychanalyste d'origine étrangère mais travaillant majoritairement avec des patients francophones. II m'est arrivé de recevoir des patients coréens qui parlaient donc coréen dans la cure. Contrairement à ma position habituelle, je me suis sentie sollicitée, presque happée par ma langue maternelle que j'entendais dans la bouche de mes patients compatriotes. Au point que je me devais de me rappeler que je suis d'abord leur analyste, leur thérapeute, pour lutter contre ce sentiment illusoire d'une unité, d'une entente immédiate que les mots coréens entendus éveillaient en moi. Je pense que l'expérience psychanalytique est une voie singulière d'accès à soi-même, dans la mesure où cette quête de soi passe par une épreuve de l'étranger externe et interne. La pratique psychanalytique montre bien combien l'illusion d'une proximité immédiate, d'une familiarité hâtive peut empêcher d'accéder à cette part d'ombre qui, sans faire partie complètement de nous-mêmes, gît et agit en chacun de nous. Comme disait Paul Ricoeur : le chemin qui mène de soi à soi est sans doute le plus long qui nécessite des détours de toutes sortes, périlleux et à la fois généreux. 